\title{
Ein Fall von regelmässig wiederkehrenden prämenstruellen Lungenblutungen.
}

\author{
Von
}

\author{
Dr. med. A. Scherer, \\ Chefarzt der Kronprinzessin Cecilie-Heilstätte.
}

Die Veröffentlichung Heimanns "Zyklischer Verlauf bei Lungentuberkulose" in Heft 1 des V. Bandes dieser Zeitschrift gibt mir Veranlassung, einen Fall von regelmässig, zusammęn mit den Menses auftretenden und jeder Behandlung Trotz bietenden Lungenblutungen der Öffentlichkeit zu übergeben. Die Hämoptoen traten zyklisch alle 24-26 Tage auf; ich selbst konnte den Fall durch sechs Monate beobachten und wurde noch weitere $4 \frac{1}{2}$ Monate, bis zum Tode der Kranken, eingehend über sie unterrichtet. Es möge zunächst ein Auszug aus der Krankengeschichte hier Platz finden:

Anamnese. B. B., Zigarettenarbeiterin aus R., verheiratet, 35 Jahre alt, anf Kosten der Landesversicherungsanstalt Posen hier untergebracht, aufgenommen am 2. Januar 1905. Der Vater soll gesund, die Mutter vor 29 Jahren an Lungentuberkulose gestorben, sieben Geschwister gesund sein, ebenso der Ehegatte und fünf Kinder; zwei Kinder sollen im ersten Lebensjabre an Darmstörungen gestorben sein. Die Kranke selbst war früher stets arbeitsfähig, ist aber seit Juli 1904 ausser Stande, ihrer Arbeit nachzugehen. Die sozialen Verhältnisse waren ziemlich günstige, abgesehen von der beruflichen Staubeinatmung. Die Patientin soll früher stets gesund gewesen sein; ihre Periode sei regelmässig, alle $3^{1}{ }_{2}$ Wochen wiederkehrend, 5-6 Tage dauernd, ziemlich stark, zuletzt vor drei Wochen aufgetreten. Sie hätte sieben normale Geburten durchgemacht, die letzte am 12. Juli 1903, die Wochenbetten seien ohne Störung verlaufen.

Anschliessend an die letzte Entbindung sei sie erkrankt mit Stechen in der rechten Seite und wenig trockenem Husten; sie hätte sich dann wohl gefühlt bis zum Juli 1904; seitdem hätte sie über Schwächegefühl, viel Husten und eitrigen Auswurf, zeitweise auch über Nachtschweisse, Fieber und starke Atemnot zu klagen. Das Körpergewicht hätte allmählich um $3 \mathrm{~kg}$ abgenommen. Mehrmals seien im letzten Halbjahre mässig starke, stets einige Tage anhaltende Lungen. 
blutungen aufgetreten; über den Zeitpunkt derselben könne sie nichts genaueres angeben.

Status praesens bei der Aufnahme: Leidliches Allgemeinbefinden; klagt über Atemnot, „Röcheln auf der Brust“, viel Husten und Auswurf, Appetitmangel, Schlaflosigkeit, wenig Schmerzen in der rechten Seite und Neigung zu Heiserkeit.

Körpergrösse $156 \mathrm{~cm}$; Gewicht 50,1 kg (leicht bekleidet); ziemlich kräftig gebauter Brustkorb von $83 / 80 \mathrm{~cm}$. Umfang, rechts weniger ausdehnungsfähig als links, schwache Muskulatur, gering entwickeltes Fettpolster, sehr blasse Haut und Schleimhäute.

Über der ganzen rechten Lunge, nach unten zu abnehmend, ziemlich starke Dämpfung, oben bronchovesikuläres, nach unten sehr stark abgeschwächtes vesiko. bronchiales Atmen und sehr reichliches feuchtes mittel- bis grossblasiges klangloses Rasseln, vermischt mit Giemen und Piepsen, entsprechend dem Unterlappen auch spärliches Reiben. Über der linken Spitze geringe Scballverkürzung und rauhes Atmen ohne Rasseln; entsprechend dem linken Unterlappen geringe Schall. verkürzung, abgeschwächtes rauhes Atmen und spärliches Knisterrasseln, vermischt mit etwas Reiben, dazwischen zuweilen grobe Rhonchi hörbar.

Herzbefund: erster Ton an der Spitze gespalten, Tätigkeit beschleunigt, aber regelmässig.

Kehlkopf: Wahre und falsche Stimmlippen leicht geschwollen und gerötet, links mehr als rechts.

Genitalien ohne krankhaften Befund. Gesteigerte Patellarreflexe; starker Nystagmus. Temperatur normal. Urin frei von Eiweiss und Zucker, Diazoreaktion negativ. Im fast rein eitrigen Auswurf massenhaft Tuberkelbazillen, verschiedene Kokken und elastische Fasern.

Verlauf: Die übliche bygienisch-diätetische Behandlung, daneben Eisenarsenikpillen.

2. bis 4. I. Normale Temperatur.

5. I. Leichter Temperaturanstieg (37,5 in ore).

6. I. Starke Haemoptoen, vier Tage anhaltend; T. bis 38,2 in ore.

8. 1. Menses.

30. I. T. 37,4 in ore.

1. II. Starke Haemoptoen, drei Tage anhaltend; T. bis 37,9 .

3. II. Menses.

25. II. T. 37,4 in ore.

27. II. Starke Haemoptoen, vier Tage dauernd; T. bis 38,4 .

28. II. Menses.

23. III. T. 37,5

24. III. Ziemlich starke Haemoptoen, drei Tage anhaltend; T. bis 38,1 .

26. III. Mensès.

18. IV. T. 37,4; prophylaktisch Morphium, dreimal subkutan je 0,01 .

19. IV. Starke Haemoptoen, fünf Tage dauernd; T. bis 38,5.

20. IV. Menses.

8. V. Febris bis 38,0 ; Herpes zoster, entsprechend dem 5. bis 9 . Interkostalnerven.

12. V. T. 38,4 ; prophylaktisch, dreimal je 0,01 Morphium subkutan; Ferripyrininbalationen.

13. V. Starke Haemoptoen, fünf Tage dauernd; T. bis 38,6 .

14. V. Menses. 
3] Ein Fall v. regelmässig wiederkehrend. prämenstruellen Lungenblutungen. 289

8. VI. T. 37,3; prophylaktisch Amylnitrit, Morphium und Abschnüren der Extremitäten.

10. VI. Menses; T. bis 37,6 .

12. VI. Måssige Haemoptoen; wiederholt am 14. VI. T. bis 37,8 .

Am 29. VI. wurde die Kranke als erwerbsunfähig entlassen. Der Lungenbefund war der gleiche, wie bei der Aufnahme; jedoch waren die Rasselgeräusche wesentlich verringert. Das Körpergewicht war trotz der häufigen und schweren Lungenblutungen auf $59,0 \mathrm{~kg}$ gestiegen.

Die Kranke hielt uns auch nach ihrer Entlassung noch durch briefliche Mitteilungen auf dem Laufenden über ihr weiteres Ergehen, bezw. liess uns durch ihren Mann über ihr Befinden berichten. Diesen Mitteilungen ist zu entnehmen, dass auch nach der Entlassung die Blutungen regelmässig wiederkehrten, das erstemal am 4. Juli, und dass sie stets ein bis zwei Tage vor dem Eintreffen der Menses auftraten. Die Kranke magerte zu Hause bald wieder stark ab, war meist ans Bett gefesselt und starb, nach Mitteilung ihres Mannes, am 15. November 1905.

Die Temperatursteigerungen hielten stets einige Tage an und hingen offenbar in erster Linie mit den Menses zusammen. Es häufen sich gerade in letzter Zeit wieder die Mitteilungen über prämenstruelle Temperatursteigerungen bei Lungentuberkulose. Ich muss gestehen, dass ich bei beginnender Tuberkulose solche Temperatursteigerungen an einem Material von nunmehr nahezu 1300 weiblichen Kranken, die ich innerhalb der letzten vier Jahre in zwei Heilstätten zu beobachten Gelegenheit hatte, nur sehr selten bemerkte; unter diesen Kranken befand sich eine grosse Anzahl solcher, an denen genaue Temperaturbestimmungen teils wegen Behandlung mit Tuberkulin, teils aus anderen Gründen Monate hindurch ununterbrochen vorgegenommen wurden, bei denen also solche prämenstruelle Temperatursteigerungen unbedingt hätten auffallen müssen. Um jedoch die etwaige Fehlerquelle, welche die wohl in den meisten Heilanstalten eingeführte und meiner Erfahrung nach bei gut luftdurchgängiger Nase vollkommen genügende Mundmessung darstellen könnte, zu beseitigen, lasse ich seit nahezu vier Monaten an einer Anzahl intelligenterer und zuverlässiger Kranker, die sich nicht nur dem klinischen Befunde, sondern auch der Anamnese nach im Anfangsstadium der Lungentuberkulose befinden, vergleichende Messungen in ore und in rekto vornehmen; bis jetzt konnte ich unter diesen - im ganzen 35 - Kranken ein prämenstruelles Fieber nur einmal beobachten; es ist mir danach nicht wahrscheinlich, dass diese prämenstruelllen Temperatursteigerungen bei beginnender Tuberkulose so sehr häufig vorkommen, wie manche Autoren annehmen, noch weniger wahrscheinlich aber, dass der Ort der Messung für das Resultat derselben ausschlaggebend sein kann, da im erwähnten Falle die Temperatursteigerung bei Mundmessung eben so deutlich zum Ausdruck kam, wie bei Rektalmessung. 
Bei vorgeschritteneren Fällen habe ich dagegen - auch bei Mundmessung - nicht selten das in Frage stehende Phänomen beobachten können. In unserem Falle war der Zusammenhang der Temperatursteigerung mit den Menses unverkennbar. Zwischen den einzelnen Perioden verhielt sich die Temperatur stets normal, mit Ausnahme der Zeit vom 8. bis 28. Mai; doch können in diesem Falle weder die Lungenblutungen noch die Menses für die Temperatursteigerungen verantwortlich gemacht werden; dieselben finden vielmehr ihre Erklärung in dem heftigen Herpes zoster. Mit einer etwa im Gefolge der Hämoptoen aufgetretenen katarrhalischen oder desquamativen Pneumonie können die Temperatursteigerungen auch nicht in Zusammenhang gebracht werden, da sie stets erst einige Tage vor den Menses auftraten und nach wenigen Tagen wieder verschwanden.

Eine Vermehrung der katarrhalischen Erscheinungen über den Lungen unmittelbar vor den Menses oder während derselben habe ich in einer nicht unerbeblichen Zahl von Fällen feststellen können. Vielfach nehmen die sonst trockenen Geräusche zu der in Frage stehenden Zeit feuchten Charakter an. In mehreren Fällen, in denen ich sonst vergeblich nach Nebengeräuschen fahndete, traten solche zurzeit der Menses deutlich auf. Ich möchte nicht unerwähnt lassen, dass ich in vereinzelten Fällen hinwiederum eine Verminderung der katarrhalischen Geräusche während der Menses konstatieren konnte. In unserem Falle gelangte ich zu keinem bestimmten Ergebnis in dieser Richtung, da ich die Kranke während der Hämoptoen oder in den auf dieselben folgenden Tagen begreiflicherweise stets nur oberflächlich untersuchen konnte.

Auffallend ist das regelmässige Auftreten der Lungenblutungen, sozusagen mit mathematischer Sicherheit stets einen bis zwei Tage vor Beginn der Menses. Die Frist vom Beginne einer Lungenblutung bis zum Eintritt der nächsten betrug stets 24-26 Tage, nur die Frist vom 13. Mai bis zum 12. Juni ist länger (30 Tage), die vom 12. Juni bis zum 24. Juli dagegen kürzer (22 Tage); die Zeit vom 13. Mai bis zum 4. Juli hinwiederum entspricht der Frist von 2 Perioden von je 26 Tagen. Ich glaube, dass für das - sit venia verbo - "verspätete" Eintreten der Lungenblutung im Juni die geübte Prophylaxe in Gestalt des Abbindens der Extremitäten mit elastischen Binden verantwortlich gemacht werden kann, zumal dies die einzige therapeutische Massnahme war, welche in diesem Falle wenigstens einigen Erfolg versprach.

In der Literatur finden sich häufig Angaben über "vikariierende" Lungenblutung, wie auch über Auftreten von Lungenblutungen wäh- 
5] Ein Fall v. regelmässig wiederkehrend prämenstruellen Lungenblutungen. 291

rend der Menses; genauere kasuistische Mitteilungen konnte ich jedoch nirgends finden. Eine wirklich „vikariierende" Lungenblutung, welche also vollkommen an Stelle der Genitalblutung getreten wäre, konnte ich niemals beobachten; wohl aber entsinne ich mich eines Falles, den ich vor etwa fünf Jahren in Behandlung hatte, in welchem während zweier aufeinanderfolgender Menstruationen Lungenblutungen auftraten. Ob dies auch bei späteren Menstruationen der Fall war, vermochte ich nicht $z u$ ermitteln. Leichtere Lungenblutungen vor Eintritt der Menses oder während derselben sind von vielen Autoren beobachtet worden. Ich selbst kann mich nur zweier weiterer Fälle erinnern, und selbst in diesen war es mir sehr zweifelhaft, ob das Blut tatsächlich aus den Lungen stammte.

Zur Unterdrückung der Lungenblutungen haben wir in unserem Falle so ziemlich unser ganzes therapeutisches Schatzkästlein ausgekramt, alles ohne Erfolg: Die Lungenblutungen hielten stets einige Tage an und ihr schliessliches Aufhören kann meines Erachtens nicht in Zusammenhang mit den therapeutischen Massnahmen gebracht werden. Die Kranke erbolte sich jedoch stets in wenigen Tagen von den schweren Blutungen. Morphium, Dionin, Kodein blieben, auch in grossen Dosen, vollkommen wirkungslos, gleichviel ob per os oder subkutan verabreicht. Gelatine, die uns in anderen Fällen von Lungenblutungen oft sehr gute Dienste tat, indem sie wenigstens einen sehr raschen Stillstand der Blutungen bewirkte, erzielte hier keinerlei Erfolg. Inhalationen von Amylnitrit vermochten die Blutungen ebensowenig zu beeinflussen, wie wochenlang fortgesetztes Inhalieren von Ferripyrin ihr Eintreten verhindern konnte. Das einzige, was wenigstens ein momentanes Sistieren der Blutungen bewirkte, war die Abschnürung sämtlicher Extremitäten mittelst elastischer Gummibänder; wir verwandten vom Medizinischen Warenhaus-Berlin gelieferte SchnallenTourniquets. Interessant erscheint es, dass gerade diese Behandlungsweise das Eintreten der Blutungen um einige Tage zu verzögern vermocbte: die Blutung, die wir am 8 . oder 9. Juni erwarten mussten, stellte sich erst am 12. Juni ein; und da die Blutungen vor- und nachher stets ganz regelmässig, um nicht zu sagen "pünktlich" eintraten, am 12. Juni aber schwächer als sonst blieben, und sich nur am 14. Juni in leichtem Grade wiederholten, ist die Wabrscheinlichkeit einer günstigen Folge dieser Massnahme wohl nicht von der Hand $\mathrm{zu}$ weisen.

Es wäre mir interessant, zu erfahren, ob auch anderwärts ähnliche Fälle beobachtet wurden; ihre Veröffentlichung erscheint mir doch recht wünschenswert, da ja nur genau beobachtete und mit genügender Ausführlichkeit beschriebene Fälle späteren Autoren ver- 
wertbares Material für Behandlung so mancher hier einschlägigen Fragen bieten. Von diesem Gesichtspunkte ging ich bei vorstehender Veröffentlichung aus, hoffend und wünschend, dass sich derartige Fälle öfters in der Literatur finden möchten, damit man auch in der Lage ist, daraus praktische Schlussfolgerungen bezüglich eines etwa bestehenden Zusammenhanges zwischen weiblichem Genitale und anderen Organen zu ziehen, was mir bei vereinzelten Fällen nicht ratsam erscheint. 